\title{
PirB restricts neuronal regeneration in developing rat brain following hypoxia-ischemia
}

\author{
HUA WANG $^{1}$, YING XIONG ${ }^{1}$ and DEZHI MU ${ }^{1,2}$ \\ ${ }^{1}$ Department of Pediatrics, West China Second University Hospital, Sichuan University, Sichuan, Chengdu 610041, P.R. China; \\ ${ }^{2}$ Department of Pediatrics, University of California, San Francisco, CA, USA
}

Received January 27, 2012; Accepted April 23, 2012

DOI: $10.3892 / \mathrm{mmr} .2012 .907$

\begin{abstract}
The inhibitors of axonal regeneration in the myelin sheath are considered to be major contributors to the lack of regeneration in the central nervous system (CNS) following hypoxic-ischemic (HI) brain damage. As well as the Nogo receptor $(\mathrm{NgR})$, the paired-immunoglobulin-like receptor $\mathrm{B}$ (PirB) is a functional receptor for the myelin inhibitors of axonal regeneration. The inhibition of PirB and $\mathrm{NgR}$ activities may block most of the inhibitory effects of myelin inhibitors on nerve regeneration. We observed the PirB protein and mRNA expression in HI-damaged rat cortical neurons using immunohistochemistry and reverse transcription-polymerase chain reaction assays. In addition, we treated the HI-damaged rat cortical neurons using PirB antibodies to observe the regeneration of injured neurons. Moreover, the Rock II activity in HI-damaged rat cortical neurons treated with PirB antibodies was observed using western blot analysis. The mRNA and protein levels of PirB increased in newborn rat cortical neurons following HI damage. Treatment with PirB antibodies is able to improve axonal regeneration following HI damage compared with normal axonal growth. Rock II activity also increased in the HI-damaged rat brain. The inhibition of PirB is therefore a potential therapeutic method to promote the regeneration of HI-damaged axons and the inhibitory signal may be transduced through the Rho-ROCK signaling pathway.
\end{abstract}

\section{Introduction}

Hypoxic-ischemic (HI) encephalopathy (HIE) is common in clinical neonatal brain damage (1). However, knowledge of HIE has always been limited. Most known treatments are symptomatic and prevent or relieve the damaging effects of hypoxia-ischemia to neurons. Prevention strategies are available

Correspondence to: Dr Dezhi Mu, Department of Pediatrics, West China Second University Hospital, Sichuan University, No. 20, The People's South Road, Sichuan, Chengdu 610041, P.R. China E-mail: dezhimu@yahoo.com

Key words: paired immunoglobulin-like receptor B, neuron regeneration, developing rat brain, hypoxia-ischemia (although limited), but currently no strategies for the support of recovery/repair are on the horizon, although hypothermia has been demonstrated in several large multicenter studies as an essential treatment. However, at present, no effective methods for promoting the regeneration of injured neurons have been identified. Therefore, new strategies for treating perinatal hypoxia-induced neuron injury are urgently needed (2).

The failure of axonal regeneration in the central nervous system (CNS) is largely attributed to the environment of the injured axons. The inhibitory activity is principally associated with CNS myelin components and the molecules in the glial scar at the lesion site. Previous studies suggested that three myelin proteins, i.e., the myelin-associated glycoprotein (MAG), Nogo-A and oligodendrocyte myelin glycoprotein (OMgp), collectively account for the majority of inhibitory activities in CNS myelin (3).

The inhibitory activities of MAG, OMgp and the extracellular domain of Nogo-A (Nogo-66) on HI-damaged neuron regeneration may be mediated by common receptor complexes consisting of the glycosylphosphatidylinositol-anchored Nogo receptor (NgR) and its signaling coreceptors (4-7). A number of studies have demonstrated that $\mathrm{NgR}$ may be involved in the pathology observed following $\mathrm{HI}$ in neonatal rats and that the inhibitory activity of $\mathrm{NgR}$ promotes axonal regeneration $(8,9)$. However, no difference has been observed in the neurite outgrowth inhibition by the myelin inhibitors in the neurons of NgR-null and wild-type mice. Moreover, no corticospinal-tract (10) regeneration has been reported. Thus, a second receptor may mediate the inhibitory signaling of myelin inhibitors.

The paired-immunoglobulin-like receptor B (PirB), a second receptor, was originally identified in the cells of the immune system and, more recently, in those of the nervous system (11). Similar to NgR, PirB binds Nogo66, MAG and OMgp. PirB is also capable of inhibiting neurite outgrowth. The combined inhibition of $\mathrm{NgR}$ and PirB results in an almost complete regeneration of damaged nerves. Moreover, neurons of NgR-null mice which have been treated with PirB antibodies partially block the axonal regeneration inhibition by Nogo66 (12). These findings strongly suggest that compounds which target PirB and NgR enhance neuronal regeneration in spinal cord injuries. However, certain studies have indicated that PirB knockout in combination with NgR blockade did not promote axonal regeneration and that blocking the function 
of PirB is not sufficient to promote axonal reorganization or functional recovery following cortical injury in vivo $(13,14)$. Therefore, it is possible that the PirB and $\mathrm{NgR}$ inhibitory signaling pathways have some unknown contacts. We aimed to investigate whether there were differences in the HI-damaged neurons of newborn rats.

In the present study, we hypothesized that the inhibition of PirB stimulates the regeneration of damaged nerves through the inhibition of the signal transduction of myelin inhibitors. PirB and $\mathrm{NgR}$ are functional receptors of myelin inhibitors and the inhibitory activity of $\mathrm{NgR}$ on axonal growth is mediated by the Rho-ROCK signaling pathways. Thus, we also hypothesized that the inhibitory activity of PirB is mediated by the Rho-ROCK signaling pathways. In the present study, we investigated the effects of PirB on axonal regeneration and explored the underlying signaling pathway in order to initiate the search for new methods that promote nerve regeneration.

\section{Materials and methods}

Animals. Two-day-old Sprague-Dawley rats were used in the experiments. The rats were obtained from the West China Medical Laboratory Animal Center and maintained in a conventional mouse facility. Protocols were approved by the Animal Care and the Guidelines of Animal Use and Protection of Sichuan University were followed. All possible efforts were made to minimize unnecessary suffering of the animals.

Primary cultures of cortical neurons. Sprague-Dawley rats were sacrificed by cervical dislocation. The cortices of rat were immersed in an ice-cold calcium- and magnesium-free Hank's Balanced Salt Solution. The tissues were digested with trypsin $\left(0.125 \%\right.$ trypsin maintained at $37^{\circ} \mathrm{C}$; HyClone Laboratories, Logan, UT, USA) for $30 \mathrm{~min}$. The trypsin solution was decanted and the tissues were washed three times with serumfree Dulbecco's minimal essential media (DMEM) $(10 \mathrm{ml})$ (HyClone Laboratories) supplemented with $20 \%$ fetal bovine serum (HyClone Laboratories). The tissues were dispersed in this medium with a screen at room temperature. The cells were placed at $1.5 \times 10^{5}$ cells $/ \mathrm{cm}^{2}$ in polylysine-coated 6 -well plates (Becton Dickinson Labware, Franklin Lakes, NJ, USA) containing DMEM supplemented with $10 \%$ heat-inactivated horse serum, $10 \%$ heat-inactivated fetal bovine serum, $2 \mathrm{mM}$ glutamine and $25 \mathrm{mM}$ glucose without antibiotics. The cultures were maintained in a humidified $5 \% \mathrm{CO}_{2}$ incubator at $37^{\circ} \mathrm{C}$. On the second day, $10 \mu \mathrm{M}$ Ara- $\mathrm{C}$ was added to the cultures to inhibit non-neuronal cell proliferation. Twice a week thereafter, half of the medium was replaced with fresh fetal bovine serum-free culture medium. The experiments on the cultured neurons were performed after 10 days.

HI neuron model. The neurons were randomly divided into four groups: negative control, deprivation of oxygen and glucose, anti-PirB antibody treatment and anti-PirB antibody-treated deprivation of oxygen and glucose. For the deprivation of oxygen and glucose group, the neurons were washed twice with sugar-free Earle's culture medium, maintained in the same medium and placed in a humidified $5 \% \mathrm{CO}_{2} / 95 \% \mathrm{~N}_{2}$ incubator at $37^{\circ} \mathrm{C}$ for $4 \mathrm{~h}$. For the anti-PirB antibody treatment group, the cells were cultured in Earle's culture medium supplemented with $50 \mu \mathrm{g} / \mathrm{ml}$ of $6 \mathrm{C} 1$, a specific anti-PirB antibody (Santa Cruz Biotechnology, Santa Cruz, CA, USA) targeting the PirB extracellular region. For the anti-PirB antibody-treated deprivation of oxygen and glucose group, the cells were cultured in Earle's culture medium supplemented with $50 \mu \mathrm{g} / \mathrm{ml}$ of $6 \mathrm{C} 1$ and placed in a humidified $5 \% \mathrm{CO}_{2} / 95 \% \mathrm{~N}_{2}$ incubator at $37^{\circ} \mathrm{C}$ for $4 \mathrm{~h}$.

Immunocytochemistry. Following treatment, the cortical neurons were fixed with $4 \%$ paraformaldehyde for $10 \mathrm{~min}$, washed three times with $0.01 \mathrm{M}$ PBS for $5 \mathrm{~min}$ and blocked with $10 \%$ goat serum dissolved in PBS at room temperature for $1 \mathrm{~h}$. The cells were then incubated together with anti-PirB antibodies overnight at $4^{\circ} \mathrm{C}$, washed three times with 0.01 M PBS for $5 \mathrm{~min}$, incubated together with goat anti-rabbit antibodies and visualized using DAB. The Metamorph software was used for capturing the images and quantification of the cell area. To measure the staining intensities, we graphically highlighted each individual neuron, including the nuclear region.

Neurite outgrowth assay. The isolated cortical neurons were placed in polylysine-coated 6 -well plates. Following the treatments, the cultures were fixed for 30 min with $4 \%$ paraformaldehyde, permeabilized with ice-cold methanol and immunostained with rabbit polyclonal antibodies against BIII-tubulin (1:400; Santa Cruz Biotechnology). The slides were viewed under an inverted fluorescence microscope. The length of the longest neurite for each $\beta$ III-tubulin-positive neuron for the first 180-200 neurons encountered upon a systematic scanning of the slide was determined using the Simple PCI image analysis program. Neurite length quantitation and statistical analyses were performed as described previously (15).

Fluorescent staining. The cells were washed with PBS and fixed with $4 \%$ paraformaldehyde at room temperature for $10 \mathrm{~min}$. After being washed twice with ice-cold PBS, the fixed cells were blocked with 5\% BSA (Sigma, St. Louis, MO, USA) at room temperature for $30 \mathrm{~min}$, followed by incubation with the primary antibodies and a specific anti-PirB antibody (Santa Cruz Biotechnology) for $1 \mathrm{~h}$ at room temperature. The cells were then treated with the secondary antibody conjugated with fluoresceinisothiocyanate (FITC; 1:100 dilution) in PBS for $1 \mathrm{~h}$ at room temperature. The images were visualized using a Zeiss Imager Z1 fluorescence microscope (Zeiss, Oberkochen, Germany).

Reverse transcription-polymerase chain reaction (RT-PCR). In a previous experiment (16), $6 \times 10^{5}$ cells from the control and HI-damaged groups were used. After being centrifuged and washed twice with $1 \mathrm{ml}$ of PBS, the cell pellets were lysed using ice-cold TRIpure TRIzol reagent. The total RNA was extracted using chloroform, isopropanol and absolute ethyl alcohol sequentially. The RNA pellets were dissolved in DEPC water. The quality and concentration of the extracted RNA were confirmed using $1 \%$ agarose gel and an ultraviolet spectrophotometer. The qualified RNA was stored at $-80^{\circ} \mathrm{C}$ until use. Reverse transcription was performed using $10 \mu \mathrm{l}$ of reaction mixture containing $1 \mu \mathrm{l}$ of $10 \mathrm{X}$ PCR buffer, $1 \mu \mathrm{l}$ of dNTP mix, $2 \mu \mathrm{l}$ of negative sense primer, $0.25 \mu \mathrm{l}$ of RNasin, $0.5 \mu \mathrm{l}$ of AMV-RT, $2.75 \mu \mathrm{l}$ of DEPC water and $2 \mu \mathrm{l}$ of the 
A

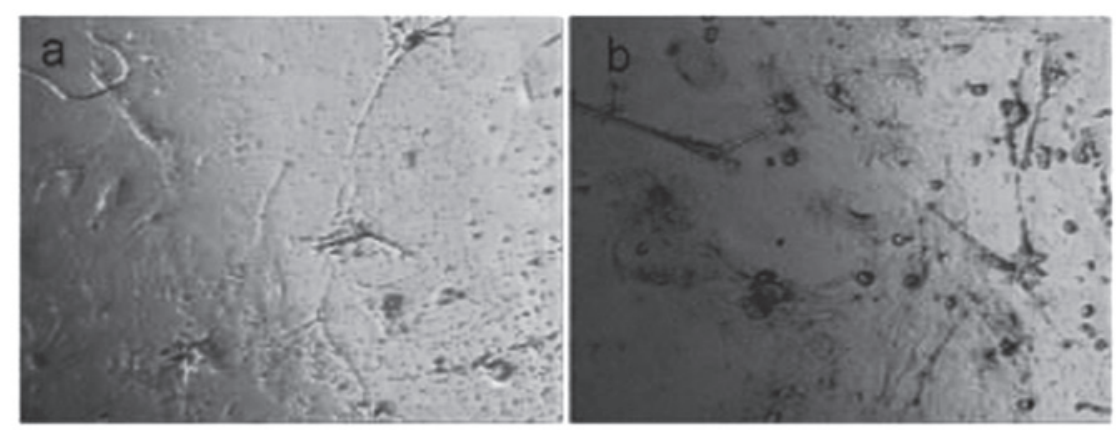

B

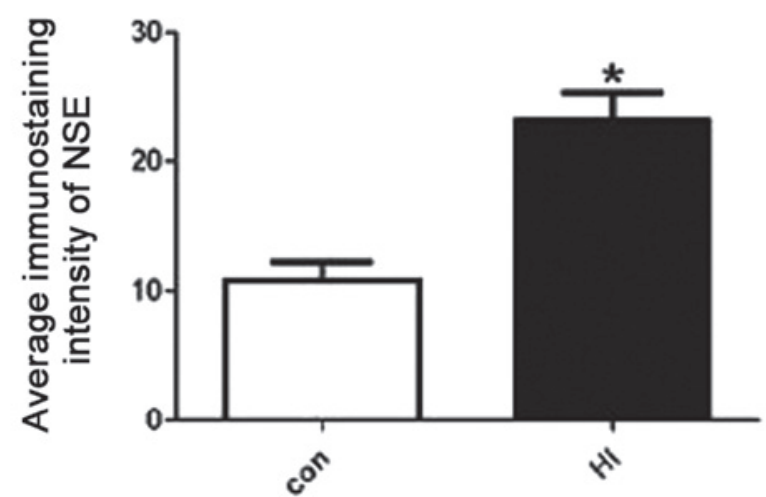

Figure 1. NSE immunostaining intensity increased in rat cortical neurons following hypoxic-ischemic damage. Immunohistochemistry assays were performed to evaluate NSE expression in newborn cortical neurons following HI injury. (A) Representative immunostaining images for NSE in cortical neurons in control (left panel) and HI-damaged neurons (right panel). (B) Student's t-test was used to compare the difference in NSE immunostaining intensity between the two groups. "P<0.05 vs. the control group $(\mathrm{n}=3)$. NSE, neuron-specific enolase; con, control; HI, hypoxic-ischemic.

extracted RNA at $42^{\circ} \mathrm{C}$ for $30 \mathrm{~min}$. The cDNA was stored at $-20^{\circ} \mathrm{C}$ until use. PCR was then performed in $50 \mu \mathrm{l}$ of reaction mixture containing $10 \mu \mathrm{l}$ of $5 \mathrm{X}$ PCR buffer, $0.25 \mu \mathrm{l}$ of Taq DNA polymerase, $0.5 \mu 1$ of targeted gene premier (sense and reverse), $0.5 \mu \mathrm{l}$ of reference gene premier (sense and reverse) and $10 \mu \mathrm{l}$ of cDNA. The thermocycler program included denaturation at $94^{\circ} \mathrm{C}$ for $2 \mathrm{~min} ; 35$ cycles at $94^{\circ} \mathrm{C}$ for $30 \mathrm{sec}$ and $58^{\circ} \mathrm{C}$ for $40 \mathrm{sec}$ for the PirB gene and $56^{\circ} \mathrm{C}$ for $40 \mathrm{sec}$ for the $\beta$-actin gene; annealing at $72^{\circ} \mathrm{C}$ for $40 \mathrm{sec}$; and a final extension at $72^{\circ} \mathrm{C}$ for $30 \mathrm{sec}$. The RT-PCR products were analyzed using $1 \%$ agarose gel electrophoresis followed by densitometric scanning. The optical density of the gene bands was analyzed using Quantity One software (Bio-Rad Laboratories, Hercules, CA, USA) and the ratios of the targeted gene products to $\beta$-actin products were calculated.

The primer pairs used for the RT-PCR with $\beta$-actin and PirB were: $\beta$-actin forward: 5'-TCCGCTGC AGACAGACTGGCCAG-3' and reverse: 5'-AGTAACAGTCC GCCTAGAAGC-3' (295bp); PirBforward:5'-TCGGGGAAAA TTCAGGAA-3' and reverse: 5'-GAGAAATCTCTAGCTT TATTT-3' (215 bp).

ROCK II kinase activation. The cortical neurons were placed in polylysine-coated $100 \mathrm{~mm}$ dishes. The cells were collected, washed twice with PBS and then lysed in immune precipitation buffer (50 mM of Tris- $\mathrm{HCl}, \mathrm{pH} 7.4,150 \mathrm{mM}$ of $\mathrm{NaCl}, 5 \mathrm{mM}$ of EDTA, $1 \%$ Nonidet P-40, $0.5 \%$ sodium deoxycholate, $0.1 \%$ SDS and proteinase inhibitors) for $1 \mathrm{~h}$ on ice. The receptor proteins were then immunoprecipitated with $50 \mu \mathrm{l}$ of protein A-agarose beads preloaded with $10 \mu \mathrm{l}$
anti-ROCK II antibodies. The immunocomplexes were suspended in 2X SDS loading buffer [125 mmol/1 Tris-HCl (pH 6.8), 20\% glycerol, $0.01 \%$ bromophenol blue, 4\% SDS and $200 \mathrm{mmol} / \mathrm{l} \mathrm{DTT}$, boiled for $5 \mathrm{~min}$ and separated using SDS polyacrylamide gel electrophoresis with $30 \mu \mathrm{g}$ for each lane. Following electrophoresis, the proteins were transferred to a PVDF membrane and infected with anti- $\beta$-actin antibodies (Santa Cruz Biotechnology) or anti-PirB antibodies (Upstate Biotechnology, Billerica, MA, USA), followed by HRP-conjugated goat anti-mouse or anti-rabbit antibodies (Santa Cruz Biotechnology). Enhanced chemiluminescence (Santa Cruz Biotechnology) was employed. The density of the protein bands was analyzed using the Quantity One software (Bio-Rad Laboratories). The ratios of the targeted protein bands to GAPDH bands were calculated.

Statistical analysis. Results were presented as the means \pm standard errors. The Student's t-test was used to determine the levels of difference among the groups. $\mathrm{P}<0.05$ was considered to indicate a statistically significant result.

\section{Results}

Cultured cortical neurons of newborn rats. The cortical neurons of newborn rats were cultured for eight days and randomly divided into two groups: control and HI-damaged. Neuron injury was determined by neuron-specific enolase immunostaining, which was predominantly and exclusively localized in neuron cytoplasm and significantly increased following $\mathrm{HI}$ treatment (Fig. 1). 
A

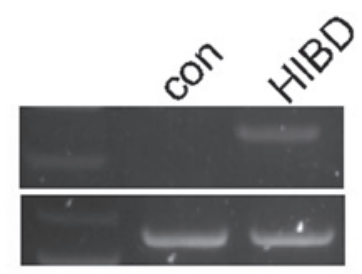

PirB

$\beta$-actin

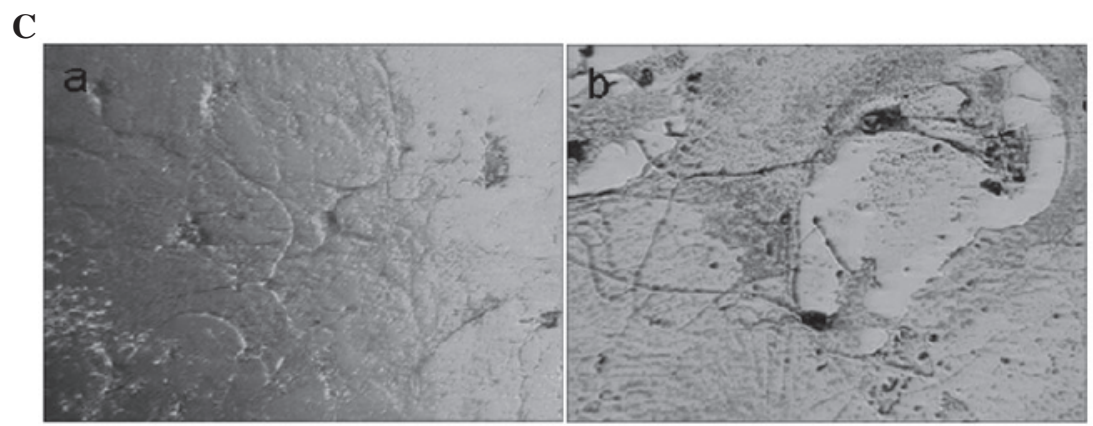

B

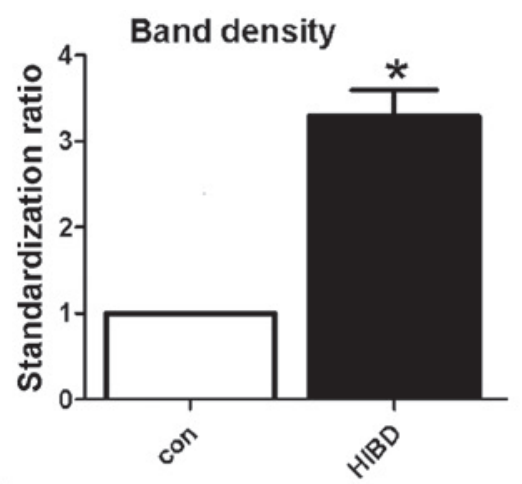

D

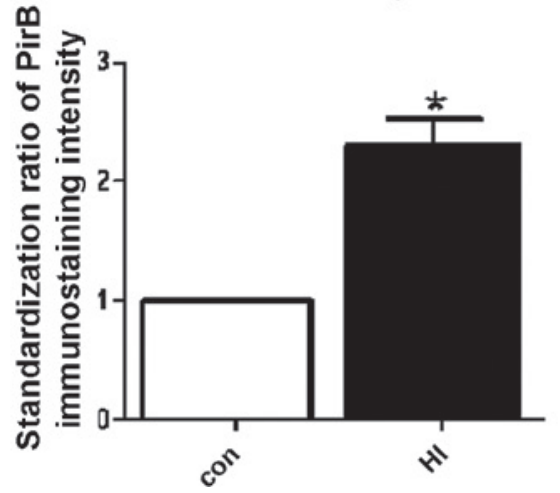

Figure 2. PirB levels increased in rat cortical neurons following hypoxic-ischemic damage. RT-PCR and immunohistochemistry were performed to evaluate PirB mRNA and protein expression in newborn cortical neurons following HI injury. (A) Representative RT-PCR images for PirB in cortical neurons in control and HI-damaged neurons; (B). Student's t-test was used to compare the difference of PirB mRNA expression between the two groups. * $<<0.05$ vs. the control group ( $\mathrm{n}=3$ ); (C) Representative immunohistochemistry images for PirB in cortical neurons in control (left panel) and HI-damaged neurons (right panel). (D) Student's t-test was used to compare the staining intensities of PirB between the two groups. * $\mathrm{P}<0.05$ vs. the control group ( $\mathrm{n}=3$ ). PirB, paired immunoglobulin-like receptor B; RT-PCR, reverse transcription-polymerase chain reaction; HI, hypoxic-ischemic; con, control; HIBD, hypoxic-ischemic brain damage.

Cortical neurons. The PirB mRNA levels of the cortical neurons were first measured using RT-PCR following HI injury. The level of PirB mRNA increased in HI-damaged cortical neurons (Fig. 2A and B). Immunocytochemistry confirmed the increased PirB protein expression in the HI-damaged group (Fig. 2C and D). These results demonstrate that the PirB mRNA and protein expression increased in the cortical neurons of newborn rats following HI damage.

Axonal regeneration. The PirB mRNA and protein expression levels increased following HI damage and findings of a previous study revealed that PirB is able to bind myelin inhibitors (12). Therefore, the blocking of PirB protein expression was investigated to determine whether it was able to enhance axonal regeneration in HI-damaged cortical neurons. The axonal length was evaluated using immunofluorescence inverted microscopy in control neurons treated with PirB antibodies, HI-damaged neurons and HI-damaged neurons treated with PirB antibodies. PirB antibody treatment was not able to promote axonal growth in normal control neurons but significantly induced regeneration in HI-damaged neurons (Fig. 3), indicating that PirB is able to improve axonal regeneration following HI damage.

ROCK II kinase expression. ROCK II plays a significant role in the inhibition of signal transduction when myelin inhibitors bind NgR. Thus, we performed a ROCK II activation assay to investigate whether ROCK II activity changed in HI-injured neurons and mediated the promoting effect of PirB antibodies on axonal regeneration. First, we observed that ROCK II expression increased following HI injury. Second, we showed that the increased ROCK II expression was blocked following the anti-PirB antibody treatment. The representative western blot analysis result is shown in Fig. 4A and the standardization ratio statistics of three tests are shown in Fig. 4B. The average intensities of the bands significantly increased in the HI-damaged group. This result suggests that ROCK II expression increased in HI-damaged cortical neurons and was blocked by PirB antibodies.

\section{Discussion}

HI brain damage (HIBD) due to peri- or postnatal asphyxia is a major cause of disability. Although various neuroprotective strategies have been studied, the options for the management of HI brain injuries are currently limited. Previous studies have revealed that the prognosis of HIE is correlated with the extent of neuronal damage. Thus, the successful regeneration of HI-injured neurons is key in HIBD treatment.

Several myelin proteins are believed to account for the inhibitory activity in CNS myelin. The most recognized of these proteins include MAG, Nogo-A and OMgp. Inhibitory signaling is transduced via their receptors, NgR and PirB. The trimeric receptor complexes stimulate the RhoA-ROCK pathway when myelin-associated inhibitors bind with Nogo-A. Nogo-A binds with NgR-P75NTR and PirB binds not only with 
A

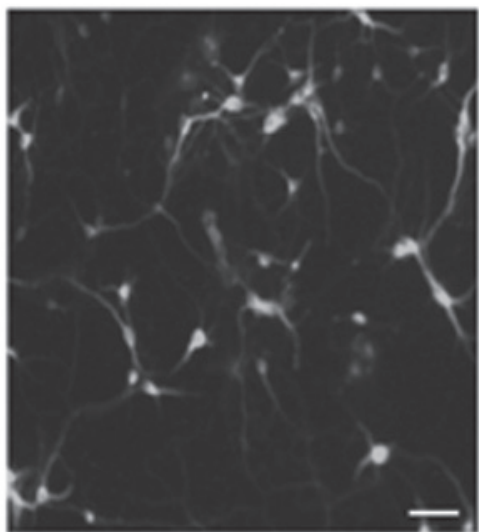

Control

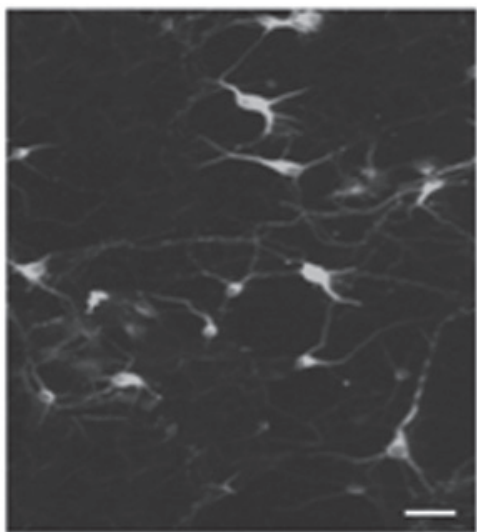

HIBD

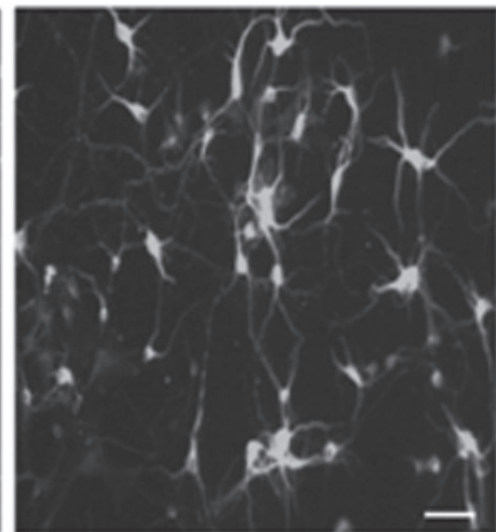

HIBD+anti PirB

$\mathbf{B}$

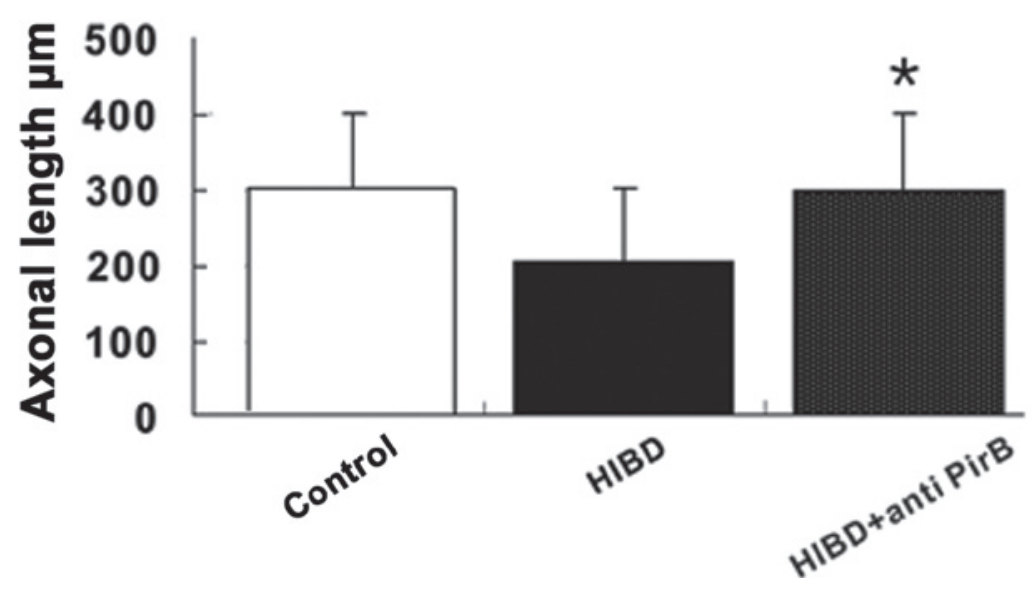

Figure 3. Blocking PirB with anti-PirB antibody enhanced axonal regeneration in rat cortical neurons following hypoxic-ischemic damage. (A) Representative immunofluorescent image of cortical neurons in control, HIBD and HIBD treated with PirB antibody group. (B) Student's t-test was used to compare the difference in axonal regeneration expression among the three groups. " $\mathrm{P}<0.05$ vs. the control group, $\mathrm{n}=3$. PirB, paired immunoglobulin-like receptor B; HIBD, hypoxic-ischemic brain damage.

A

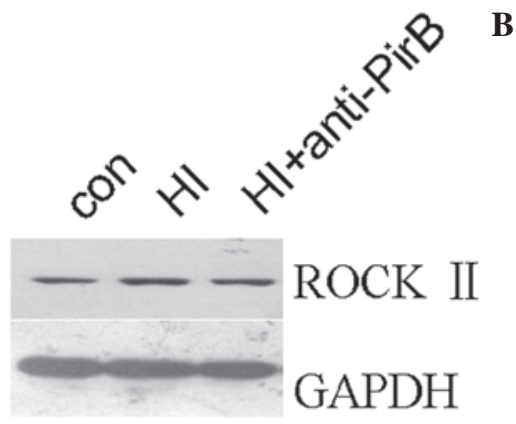

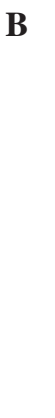

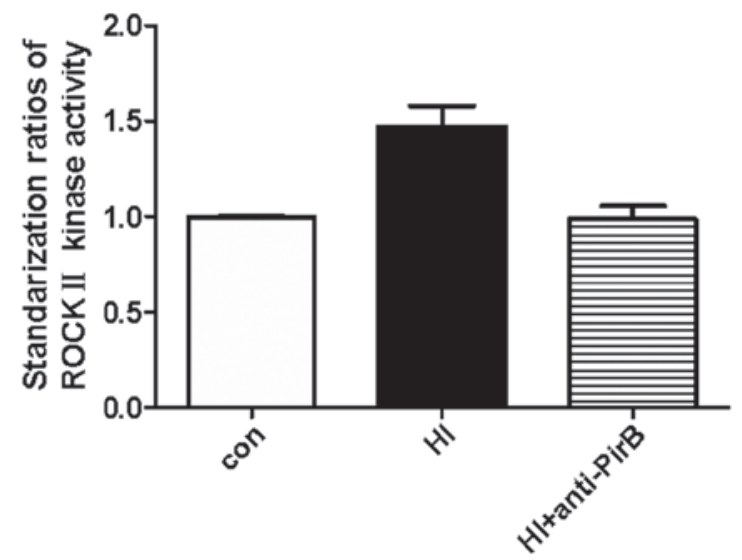

Figure 4. Enhanced ROCK II kinase activity in rat cortical neurons following hypoxic-ischemic injury could be blocked by anti-PirB antibody. Immunoprecipitation and western blot analysis were performed to evaluate ROCK II kinase activity in newborn cortical neurons following HI injury. (A) Representative western blot analysis images for ROCK II in cortical neurons in control, HI-damaged and HI+anti-PirB groups. (B) Student's t-test was used to compare the difference in ROCK II kinase activity among the three groups. ${ }^{*} \mathrm{P}<0.05$ vs. the control group ( $\mathrm{n}=3$ ). PirB, paired immunoglobulin-like receptor B; HI, hypoxic-ischemic; con, control.

Nogo66 but also with MAG and OMgp with, at least for MAG, the same affinity as that when it binds with NgR-P75NTR (10).

The identification of PirB as a receptor for three major myelin inhibitors of regeneration not only expands our understanding but also adds to the complexity of preventing axonal regeneration following brain injury. Thus, PirB is also a possible target for therapeutic intervention to promote in vivo regeneration. However, certain studies have indicated that PirB knockout in combination with NgR blockade did not promote axonal regeneration and that blocking the function of PirB is 
not sufficient to promote axonal reorganization or functional recovery following cortical injury in vivo $(13,14)$. Therefore, it is possible that the PirB and $\mathrm{NgR}$ inhibitory signaling pathways have some unknown contacts and PirB may have a role in inhibiting axonal regeneration in specific PirB-expressing neurons in the CNS.

In the present study, we first examined PirB expression in the cortical neurons of newborn rats following HI injury. Then, we blocked the PirB protein expression using PirB antibodies to determine whether it was possible to improve axonal regeneration.

The results of the immunohistochemistry assay using a specific antibody targeting the PirB intracellular segment demonstrated that the distribution and content of PirB increased in newborn rats following HIBD. Most of the PirB-positive neurons were localized in the cerebral cortex, hippocampus and cerebellum regions in which the $\mathrm{MHC1}$ mRNA and protein were mainly located.

PirB, with a molecular weight of $130 \mathrm{kDa}$, was identified in the immunoprecipitate from the brains of newborn rats. This molecular weight is consistent with that of the PirB found in the immune system. Our previous results demonstrate that PirB protein expression significantly increased in the brain tissues of newborn rats following HIBD using western blot analysis. RT-PCR and immunohistochemistry also revealed that PirB mRNA and the protein expression increased in the cortical neurons of newborn rats following HI injury. The anti-PirB antibodies specifically targeting the PirB extracellular region promoted the axonal regeneration of the cortical neurons following $\mathrm{HI}$ injury, indicating that the inhibition of PirB is a potential therapeutic tool for improving axonal regeneration following HIBD.

As a functional receptor for myelin inhibitors, the underlying signaling pathways that transduce the inhibitory signal after PirB binds with the myelin inhibitors need to be identified. Considering that Rho-ROCK plays a significant role in $\mathrm{NgR}$-mediated inhibitory effects and that Rho expression and activity were elevated following HIBD, we hypothesized that the PirB-mediated inhibitory effects are realized through the Rho-ROCK signaling pathway. ROCK is a serine/threonine protein kinase and two isoforms of ROCK encoded by two different genes have been described: ROCK I (also known as ROK $\beta$ or p160 ROCK) and ROCK II (also known as ROK $\alpha$ ). ROCK II is preferentially expressed in the brain (17), whereas ROCK I has the highest expression levels in non-neuronal tissues. Our results demonstrate that ROCK II activity increased in the cortical neurons of newborn rats following HI damage and this increase could be inhibited by anti-PirB antibodies. This finding suggests that ROCK II is regulated by PirB and that Rho-ROCK may be the signaling pathway that transduces inhibitory signaling from PirB to the intracellular part of neurons.

\section{Acknowledgements}

This study was supported by the grants from the National Natural Scientific Fundation of China (No. 81070521; 30822039).

\section{References}

1. Roland EH and Hill A: Clinical aspects of perinatal hypoxicischemic brain injury. Semin Pediatr Neurol 2: 57-71, 1995.

2. Lai MC and Yang SN: Perinatal hypoxic-ischemic encephalopathy. J Biomed Biotechnol 2011: 609813, 2011.

3. Schwab ME: Nogo and axon regeneration. Curr Opin Neurobiol 14: $118-124,2004$.

4. Fournier AE, GrandPre T and Strittmatter SM: Identification of a receptor mediating Nogo-66 inhibition of axonal regeneration. Nature 409: 341-346, 2001.

5. Wang KC, Koprivica V, Kim JA, Sivasankaran R, Guo Y, Neve RL and He Z: Oligodendrocyte-myelin glycoprotein is a Nogo receptor ligand that inhibits neurite outgrowth. Nature 417: 941-944, 2002.

6. Domeniconi M, Cao Z, Spencer T, et al: Myelin-associated glycoprotein interacts with the Nogo66 receptor to inhibit neurite outgrowth. Neuron 35: 283-290, 2002.

7. Shao Z, Browning JL, Lee X, et al: TAJ/TROY, an orphan TNF receptor family member, binds Nogo-66 receptor 1 and regulates axonal regeneration. Neuron 45: 353-359, 2005.

8. Wang H, Yao YJ and Mu DZ: Expression of Nogo-A and NgR in the developing rat brain after hypoxia-ischemia. Brain Res 1114: 212-220, 2006.

9. GrandPré T, Li S and Strittmatter SM: Nogo-66 receptor antagonist peptide promotes axonal regeneration. Nature 417: 547-551, 2002.

10. Zheng B, Atwal J, Ho C, He XL, Garcia KC, Steward O and Tessier-Lavigne M: Genetic deletion of the Nogo receptor does not reduce neurite inhibition in vitro or promote corticospinal tract regeneration in vivo. Proc Natl Acad Sci USA 102: 1205-1210, 2005.

11. Syken J, GrandPre T, Kanold PO and Shatz CJ: PirB restricts ocular-dominance plasticity in visual cortex. Science 313: 1795-1800, 2006.

12. Atwal JK, Pinkston-Gosse J, Syken J, Stawicki S, Wu Y, Shatz C and Tessier-Lavigne M: PirB is a functional receptor for myelin inhibitors of axonal regeneration. Science 322: 867-870, 2008.

13. Nakamura Y, Fujita Y, Ueno M, Takai T and Yamashita T: Paired immunoglobulin-like receptor B knockout does not enhance axonal regeneration or locomotor recovery after spinal cord injury. J Biol Chem 286: 1876-1883, 2011.

14. Omoto S, Ueno M, Mochio S, Takai T and Yamashita T: Genetic deletion of paired immunoglobulin-like receptor B does not promote axonal plasticity or functional recovery after traumatic brain injury. J Neurosci 30: 13045-13052, 2010.

15. Cohen-Cory S and Fraser SE: Effects of brain-derived neurotrophic factor on optic axon branching and remodelling in vivo. Nature 378: 192-196, 1995.

16. Cohen-Cory S and Fraser SE: Effects of brain-derived neurotrophic factor on optic axon branching and remodelling in vivo. Nature 378: 192-196, 1995.

17. Hashimoto R, Nakamura Y, Kosako H, Amano M, Kaibuchi K, Inagaki $\mathrm{M}$ and Takeda M: Distribution of Rho-kinase in the bovine brain. Biochem Biophys Res Commun 263: 575-579, 1999. 